\title{
Physical and electrical characteristics of supramolecular polymer films based on guanosine derivatives modified with tetrathiafulvalene moiety
}

\begin{abstract}
Seong Jib Choi, Junpei Kuwabara and Takaki Kanbara
Supramolecular films consisting of guanosine derivatives modified with a tetrathiafulvalene (TTF) moiety have been prepared. The hydrogen bonding network of the guanosine unit enables the formation of a robust and self-supporting cast film by a solution process. Differential scanning calorimetry and dynamic mechanical analysis (DMA) revealed that the self-supporting films were mechanically flexible and their glass transition temperature was lower than room temperature. The results of the DMA also showed the viscoelastic properties of the films at room temperature. The physical properties of the films of the derivatives depended on both the length of the alkyl chains and the structure of their terminal group. The chemical oxidation of the derivatives resulted in the formation of the absorption band due to the radical cationic TTF moiety. The composite film of the derivatives with $7,7,8,8$,-tetracyanoquinodimethane exhibited electrical conductivity.
\end{abstract}

Polymer Journal (2012) 44, 946-951; doi:10.1038/pj.2012.47; published online 4 April 2012

Keywords: 2-deoxyguanosine; electrical conductivity; hydrogen bond; supramolecular polymer; tetrathiafulvalene

\section{INTRODUCTION}

Tetrathiafulvalene (TTF) derivatives are well known organic $\pi$-donors. These molecules have received immense attention as electrically conducting materials. ${ }^{1,2}$ TTF derivatives show electrical conductivity in the presence of oxidants in their crystalline state because of efficient interactions between $\pi$-orbitals leading to high mobility of charge carriers. ${ }^{3}$ However, the charge-transfer (CT) complexes of TTF tend to be brittle and are therefore not suited to fabrication of practical macroscale devices. Incorporation of TTF in a polymer structure is one of the simple strategies being investigated for solving this problem. Although various polymers with TTF moieties have been synthesized and characterized in terms of their electrical properties, most of these polymers exhibited low solubility or low electrical conductivity. ${ }^{4-11}$ Self-organization of TTF using supramolecular interactions is regarded as another approach to produce onedimensional alignment of TTF with high electrical conductivity. The advantages of the supramolecular approach are the achievement of high solubility in the dispersed molecular state and highly ordered structure formation in the assembled solid state. There are several examples in material science of gel-like soft materials at the micro or nano scale, based on TTF molecules made via supramolecular interactions. ${ }^{12-17}$ To obtain an increase in scale for applications, stronger intermolecular interactions are required in the structures of supramolecules with TTF. In previous work, ${ }^{18}$ we have investigated guanosine derivatives containing a TTF moiety. Guanosine is a good candidate for forming a supramolecular polymer as it is capable of forming multiple hydrogen bonds with three hydrogen bonding acceptors and donors. ${ }^{19-22}$ In addition, these hydrogen bonding networks tend to align the TTF moieties. The derivatives were expected to show polymeric properties because of the hydrogen bonding network, and electrical conductivity based on the CT complexes. In this work, we report the systematic investigation of the physical and electrical properties of these supramolecular films.

\section{EXPERIMENTAL PROCEDURE}

Materials

The reagents were purchased from Tokyo Chemical Industry Co., Ltd (Tokyo, Japan), Kanto Chemical Co., INC. (Tokyo, Japan) and Wako Pure Chemica Industries, Ltd (Osaka, Japan), and were used without further purification. Anhydrous solvents were purchased from Kanto Chemical Co., INC. Column chromatography was carried out by using silica gel 60 (Kanto Chemical Co., INC., $40-100 \mu \mathrm{m}$, neutral). The guanosine derivatives 1-3 were synthesized by literature procedures. ${ }^{18}$

\section{Synthesis of 4}

$3^{\prime}, 5^{\prime}$-Bis-O-hexyloxy-di-iso-propylsilyl-2'-deoxyguanosine. To a solution of triethylamine $(0.27 \mathrm{ml}, 2.0 \mathrm{mmol})$ and diisopropyldichlorosilane $(0.72 \mathrm{ml}$, $4.0 \mathrm{mmol})$ in $\mathrm{CH}_{2} \mathrm{Cl}_{2}(15 \mathrm{ml})$ was added a solution of 1-hexanol $(0.25 \mathrm{ml}$, $2.0 \mathrm{mmol})$ in $\mathrm{CH}_{2} \mathrm{Cl}_{2}(4.0 \mathrm{ml})$ at $0{ }^{\circ} \mathrm{C}$. After the mixture was stirred for $3 \mathrm{~h}$ at $40{ }^{\circ} \mathrm{C}$, the volatiles were evaporated in vacuo. To the remaining crude product was added DMF $(3.0 \mathrm{ml})$, imidazole $(0.14 \mathrm{~g}, 2.0 \mathrm{mmol})$ and $2^{\prime}$-deoxyguanosine $(0.13 \mathrm{~g}, 0.50 \mathrm{mmol})$. The mixture was stirred overnight at room temperature. The resulting solution was quenched with water. The organic material was 
extracted with ethyl acetate and dried over $\mathrm{MgSO}_{4}$ and concentrated in vacuo. The residue was purified by column chromatography on silica gel using ethanol/ethyl acetate $(1: 10 \mathrm{v} / \mathrm{v})$ as an eluent $(0.26 \mathrm{~g}, 75 \%)$. Proton nuclear magnetic resonance spectroscopy $\left({ }^{1} \mathrm{H} \mathrm{NMR}\right)\left(270 \mathrm{MHz}, \mathrm{CDCl}_{3}\right): \delta 12.05$ (br, $1 \mathrm{H}), 7.79,(\mathrm{~s}, 1 \mathrm{H}), 6.30-6.26(\mathrm{~m}, 1 \mathrm{H}), 6.07$ (br, $2 \mathrm{H}), 4.80-4.79$ (m, $1 \mathrm{H})$, 4.10-4.09 (m, 1 H), 3.95-3.92 (m, 2 H), 3.77-3.68 (m, 4 H), 2.63-2.53 (m, $1 \mathrm{H}), 2.48-2.40(\mathrm{~m}, 1 \mathrm{H}), 1.58-1.48(\mathrm{~m}, 4 \mathrm{H}), 1.29-1.26(\mathrm{~m}, 12 \mathrm{H}), 1.07-1.04$ $(\mathrm{m}, 28 \mathrm{H}), 0.90-0.83(\mathrm{~m}, 6 \mathrm{H}) ;{ }^{13} \mathrm{C}\left\{{ }^{1} \mathrm{H}\right\}$ NMR $\left(68 \mathrm{MHz}, \mathrm{CDCl}_{3}\right): \delta 159.1$, $153.3,151.5,135.6,117.4,88.0,83.7,72.2,63.2,63.2,62.8,41.3,32.9,32.8$, $31.7,25.5,22.7,17.5,17.4,14.1,12.2,12.1$; electrospray ionization-mass spectrometry: $m / z$ calculated for $\mathrm{C}_{34} \mathrm{H}_{66} \mathrm{~N}_{5} \mathrm{O}_{6} \mathrm{Si}_{2}[M+\mathrm{H}]^{+}: 696.5$; found: 696.5; elemental analysis: calculated (\%) for $\mathrm{C}_{34} \mathrm{H}_{65} \mathrm{~N}_{5} \mathrm{O}_{6} \mathrm{Si}_{2}$ : C 58.67, $\mathrm{H} 9.41$, N 10.06\%; found: C 58.36, H 9.22, N 9.67\%

\section{Measurements}

NMR spectra were recorded on a JEOL EX-270 NMR spectrometer (Jeol, Tokyo, Japan). Elemental analyses were carried out with a Perkin-Elmer 2400CHN instrument (Perkin-Elmer, Waltham, MA, USA). Electrospray ionization-mass spectra were recorded on an Applied Biosystems QStar Pulsar $i$ spectrometer (Applied Biosystems, Carlsbad, CA, USA). Absorption spectra were recorded using a Shimadzu UV-3100 spectrophotometer (Shimadzu, Kyoto, Japan). Indium tin oxide-coated electrode $\left(6.5-7.1 \Omega \mathrm{sq}^{-1}, 2000 \AA\right.$ ) was purchased from GEOMATIC (Kanagawa, Japan). The electrical conductivities of cast films were measured by using a two-probe method at room temperature with indium tin oxide electrode fabricated by wet etching. X-ray diffraction patterns were recorded on a Philips X'Pert MRD (Philips, Amsterdam, The Netherlands) with $\mathrm{CuK} \alpha$ radiation $(\lambda=1.542 \AA)$ at a scanning rate of $0.088^{\circ} \mathrm{s}^{-1}$ in $2 \theta$ ranging $2.5-60^{\circ}$ at room temperature. The self-supporting films were fabricated by a solvent-casting method using $0.1 \mathrm{M}$ solution in tetrahydrofuran on a glass plate $(18 \times 18 \mathrm{~mm})$. The thermal properties were measured on SII EXSTAR6200 differential scanning calorimetry (DSC) instruments (Seiko Instruments Inc., Chiba, Japan) and a EXSTAR TG/DTA6300 instrument (Seiko Instruments Inc.). The tensile strength of the self-supporting film was measured by a RSA-G2 (TA Instruments, New Castle, DE, USA) at $25^{\circ} \mathrm{C}$. The dynamic mechanical measurements were conducted using a Q800 Dynamic Mechanical Analyzer (DMA) (TA Instruments) at single-frequency scanning mode of $1 \mathrm{~Hz}$ and a cooling rate of $1^{\circ} \mathrm{C} \mathrm{min}{ }^{-1}$ over a temperature range from 25 to $-100{ }^{\circ} \mathrm{C}$. Amplitude of $0.1 \%$ was used in the film $(14.83(\mathrm{~L}) \mathrm{mm} \times 4.00(\mathrm{~W}) \mathrm{mm} \times 0.07(\mathrm{~T}) \mathrm{mm})$

\section{RESULTS AND DISCUSSION}

Derivatives 1, 2 and $\mathbf{3}$ were synthesized according to literature procedures (Figure 1). ${ }^{18}$ Derivative 4 without a TTF moiety was synthesized as a control in order to evaluate the effect of the TTF moiety in terms of self-assembly and physical properties.

The syntheses of the derivatives proceeded efficiently without the need for a protecting process on the guanine moiety and chemical oxidation of the TTF moiety was seen not to occur during the reactions. The hydrogen bonding of the derivatives was confirmed by proton ${ }^{1} \mathrm{H}$ NMR. The ${ }^{1} \mathrm{H}$ NMR signals of $\mathrm{NH}$ and $\mathrm{NH}_{2}$ in the guanosine moiety shifted downfield with increasing concentration or decreasing temperature. Similar shifts were observed for all of the derivatives. These tendencies were consistent with those of reported hydrogen bond networks of guanosine derivatives in the solution state. $^{14,18,23,24}$ Owing to the hydrogen bonding network, it was hypothesized that it would be possible to fabricate a polymer-like film from these materials. ${ }^{23}$ Indeed, the fabrication of macroscale cast films was achieved by a solvent-casting method on a glass plate. The cast film was fabricated from a 0.1-M solution of derivatives 1-4 in tetrahydrofuran or chloroform on a glass plate. On the basis of the NMR observations, the formation of the films was attributed to the hydrogen bonding network of the guanine units. ${ }^{23-26}$

Figure 2 shows photographs of the films synthesized from guanosine derivatives $\mathbf{1 - 4}$. It can be seen that the films possessed adequate strength to form self-supporting structures. The film of derivative 1 was stiff, whereas the films of derivatives 2, $\mathbf{3}$ and $\mathbf{4}$ were flexible. These observations suggest that the length of the alkyl chains affects the physical properties of the films.

\section{Thermodynamical and mechanical properties}

The thermal stability of derivatives 1-4 was evaluated by thermogravimetry analysis. Below $200{ }^{\circ} \mathrm{C}$, no loss of weight was observed, which indicated that solvent molecules were not contained in the solid state (Supplementary Figure S1). The elemental analyses, DSC, and ${ }^{1} \mathrm{H}$ NMR spectroscopy also suggested that the derivatives did not contain solvent molecules. Therefore, the self-supporting films appeared to be constructed by hydrogen bonding without the assistance or incorporation of solvent molecules. The $5 \%$ weight loss temperatures $\left(T_{\mathrm{d} 5 \%}\right)$ of derivatives 1-4 were $225.4^{\circ} \mathrm{C}, 241.1^{\circ} \mathrm{C}$, $256.4^{\circ} \mathrm{C}$ and $249.9^{\circ} \mathrm{C}$, respectively (Table 1 ). The thermal stability of the TTF-substituted derivatives increased with the alkyl chain length $(1<\mathbf{2}<\mathbf{3})$. High thermal stability of the derivative with the long alky chains is attributed to a high content of heat-resistant alkyl chains in molecular weight. The thermal stability of derivative 4 was comparable to that of derivative 2 , which has an alkyl chain of similar length. To obtain detailed information on the thermal properties of the derivatives, DSC measurements were carried out on the basis of the thermogravimetry analysis data (Supplementary Figure S2). Table 1 summarizes the thermal properties of the derivatives 1-4.

In the first DSC heating run, derivatives 1-4 exhibited only endothermic peaks at $122^{\circ} \mathrm{C}, 95.1^{\circ} \mathrm{C}, 75.2^{\circ} \mathrm{C}$ and $162{ }^{\circ} \mathrm{C}$, respectively. These peaks were assigned to the solid-to-liquid phase change of the substances $\left(T_{\mathrm{m}}\right)$. The curves obtained from the second DSC heating run of derivatives 1-4 showed gentle slopes at $-1.2^{\circ} \mathrm{C},-4.6{ }^{\circ} \mathrm{C}$, $-21^{\circ} \mathrm{C}$ and $-52^{\circ} \mathrm{C}$, respectively. These slopes were attributed to a glass transition $\left(T_{\mathrm{g}}\right)$. In addition, exothermic peaks were observed at around $10^{\circ} \mathrm{C}$, which was considered to be the crystallization temperatures $\left(T_{\mathrm{c}}\right)$ of these derivatives. The existence of $T_{\mathrm{c}} \mathrm{s}$ indicates that the derivatives have both the amorphous and crystalline phases that are often observed in conventional polymers. As $T_{\mathrm{g}}$ and $T_{\mathrm{c}}$ are lower than room temperature, derivatives 1-4 seem to be polymerlike at this temperature. The $T_{\mathrm{m}}$ and $T_{\mathrm{g}}$ of the TTF-substituted derivatives shifted to lower temperatures with increasing alkyl chain length. These results suggest that the thermal properties of the derivatives depend on alkyl chain length. The $T_{\mathrm{g}}$ of derivative 2 was higher than that of derivative 4 even though they have alkyl chains of a similar length. This was attributed to the presence of the large TTF terminal unit. ${ }^{27}$ In repeated DSC heating runs, the $T_{\mathrm{m}} \mathrm{s}$ of the derivatives shifted to lower temperatures. As the ${ }^{1} \mathrm{H}$ NMR spectra showed no apparent decomposition of the derivatives after DSC analysis, the shifts of $T_{\mathrm{m}}$ were probably caused by changes in intermolecular interactions in the film state via thermal treatments. $^{21}$

To evaluate the polymer-like properties of the materials, the tensile strength of the self-supporting films of derivatives 2-4 was measured using the tensile testing mode of DMA. Table 2 summarizes the mechanical properties. The tensile strength of derivative 1 could not be measured because of the brittleness of its film. The tensile strengths of derivatives $2-4$ were $1.2,0.19$ and $2.5 \mathrm{MPa}$ at the breaking point with elongation percentages at $25^{\circ} \mathrm{C}$ of $23 \%, 20 \%$ and $2.7 \%$, respectively (Table 2). The decrease in tensile strength of the selfsupporting film of $\mathbf{3}$ compared with that of $\mathbf{2}$ is attributed to the longer alkyl chain. However, the tensile strength of derivative 4 was larger than that of derivative $\mathbf{2}$ even though they had alkyl chains of a similar length. These results indicate that the absence of the TTF 


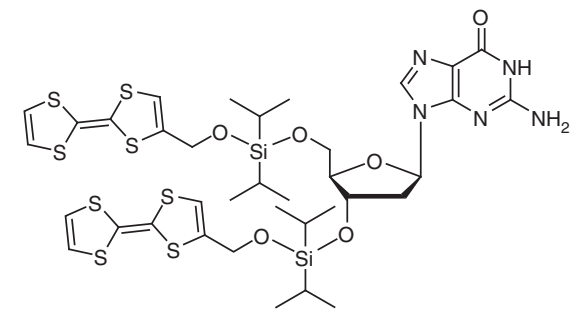

1

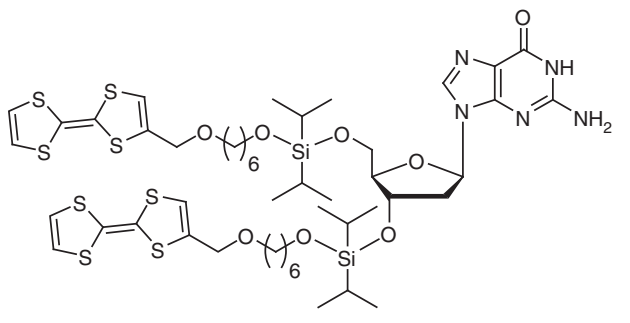

2

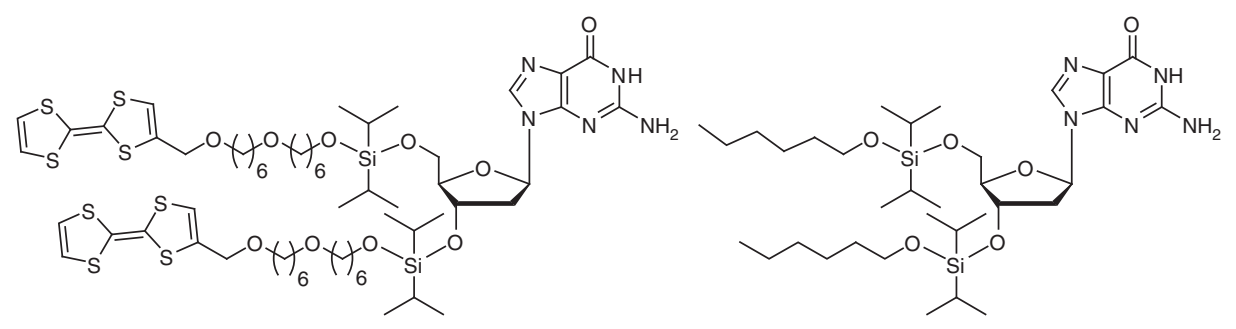

3

Figure 1 Structure of the guanosine derivatives 1-4.
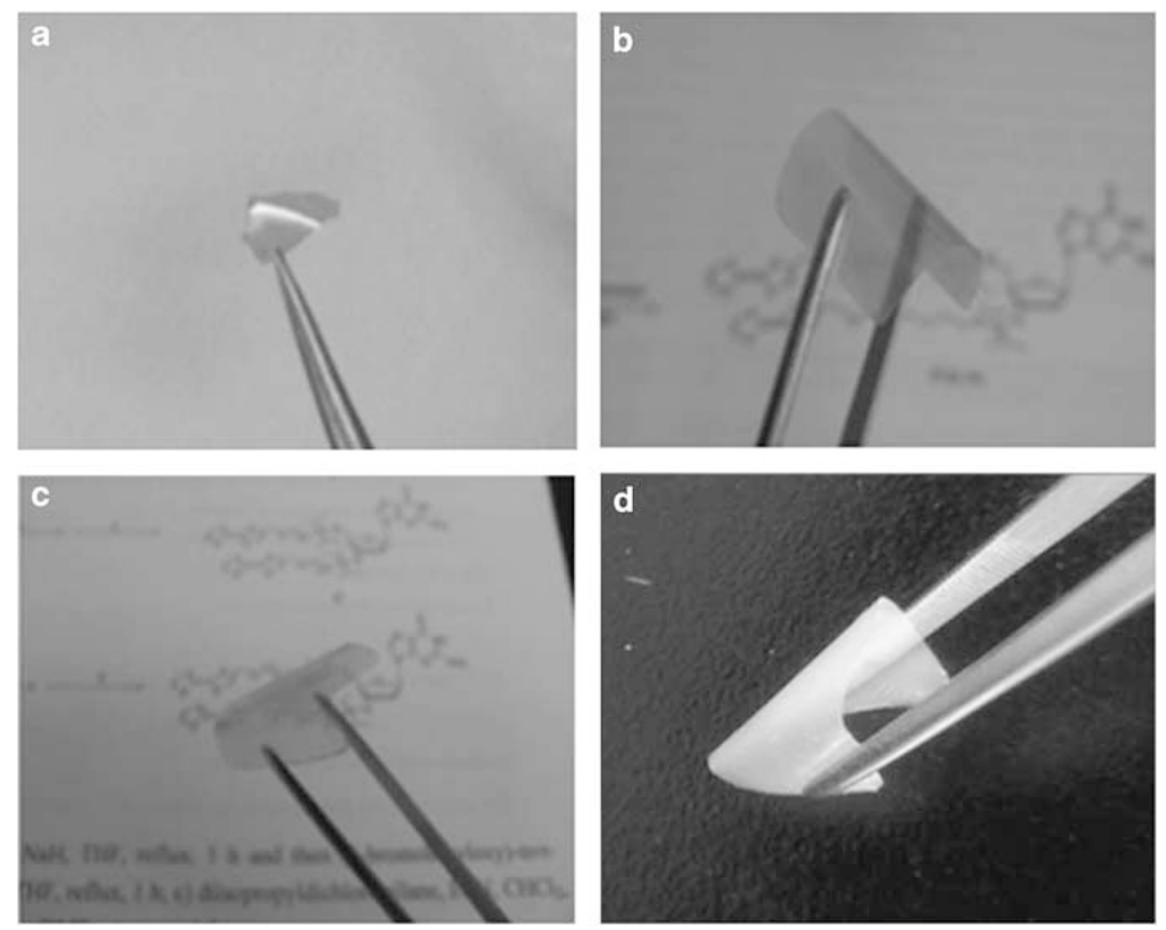

Figure 2 Self-supporting films of the derivatives (a) 1 , (b) $\mathbf{2}$, (c) 3 and (d) 4 ; the size of all derivatives is $18 \times 18 \mathrm{~mm}$, except for derivative 1 . A full color version of this figure is available at Polymer Journal online.

moiety strengthened the intermolecular interaction between the alkyl chains of derivative 4 .

We also investigated the polymer-like properties of the selfsupporting films using the dynamic modulus mode of DMA (Figure 3). The DMA curves of the derivatives were similar to those of representative viscoelastic polymers. ${ }^{28-30}$

The storage modulus $\left(E^{\prime}\right)$ of all of the derivatives decreased with increasing temperature and was higher than the loss modulus $\left(E^{\prime \prime}\right)$ at all temperatures tested (Supplementary Figure S3). These results suggest that the physical properties of the self-supporting films are solid-like. ${ }^{29}$ As the $E^{\prime}$ of derivative 4 (184 MPa) was larger than that of derivative 2, (8.28 MPa) even though their alkyl chains were similar in length, it appears that the van der Waals forces between the simple alkyl chains were stronger than those between the alkyl chains with TTF as the terminal group at $25^{\circ} \mathrm{C}$. The maximum point of a tan delta curve $\left(E^{\prime \prime} / E^{\prime}\right)$ indicates the $T_{\mathrm{g}}{ }^{28.29}$ From the maximum points of the tan delta listed in Table 2, the $T_{\mathrm{g}}$ values of derivatives 2-4 were estimated to be $-3.7^{\circ} \mathrm{C},-19^{\circ} \mathrm{C}$ and $-51{ }^{\circ} \mathrm{C}$, respectively. These values of $T_{\mathrm{g}}$ were consistent with those obtained using DSC. As the values of tan delta indicated fluidity of the films, the materials 
Table 1 The thermal properties of derivatives $1-4$

\begin{tabular}{lcccccc}
\hline Derivative & $\begin{array}{c}M W \\
\left(g \cdot \mathrm{mol}^{-1}\right)\end{array}$ & $\begin{array}{c}\mathrm{T}_{d 5 \%} \\
\left({ }^{\circ} \mathrm{C}\right)^{a}\end{array}$ & $\begin{array}{c}\mathrm{T}_{g 2 n d} \\
\left({ }^{\circ} \mathrm{C}\right)^{b}\end{array}$ & $\begin{array}{c}\mathrm{T}_{c} \\
\left({ }^{\mathrm{C} C}\right)^{\mathrm{c}}\end{array}$ & $\begin{array}{c}\mathrm{T}_{\text {m1st }} \\
\left({ }^{\circ} \mathrm{C}\right)^{d}\end{array}$ & $\begin{array}{c}\mathrm{T}_{\text {m3rd }} \\
\left({ }^{\circ} \mathrm{C}\right)^{e}\end{array}$ \\
\hline $\mathbf{1}$ & 960.5 & 225.4 & -1.2 & 6.9 & 123 & 79.5 \\
$\mathbf{2}$ & 1161 & 241.1 & -4.6 & 17 & 95.1 & 76.2 \\
$\mathbf{3}$ & 1361 & 256.4 & -21 & 21 & 75.2 & 74.7 \\
$\mathbf{4}$ & 696.1 & 249.9 & -52 & 15 & 162 & 126 \\
\hline
\end{tabular}

Abbreviations: DSC, differential scanning calorimetry; MW, molecular weight. ${ }^{a}$ Temperature for $5.0 \%$ thermal decomposition obtained by thermogravimetry analysis (heating rate of $10^{\circ} \mathrm{C} \mathrm{min}^{-1}$, argon atmosphere).

bGlass transition temperature obtained by DSC curve of second heating run.

cPhase transition temperature obtained by DSC of second heating run.

'Melting point on the fist heating run of DSC.

Melting point on the third heating run of DSC.

Table 2 The mechanical properties of the films of derivatives 2-4

\begin{tabular}{lcccccc}
\hline Derivative & $\sigma_{E}(\mathrm{MPa})^{a}$ & $\mathrm{E}(\%)^{b}$ & $\mathrm{E}^{\prime}(\mathrm{MPa})^{c}$ & $\mathrm{E}^{\prime \prime}(\mathrm{MPa})^{d}$ & $\mathrm{~T}_{g}\left({ }^{\circ} \mathrm{C}\right)^{e}$ & $\operatorname{Tan}^{f}$ \\
\hline $\mathbf{2}$ & 1.2 & 23 & 8.28 & 1.49 & -3.7 & 0.6 \\
$\mathbf{3}$ & 0.19 & 20 & 3.19 & 0.599 & -19 & 0.7 \\
$\mathbf{4}$ & 2.5 & 2.7 & 184 & 4.69 & -51 & 0.2 \\
\hline
\end{tabular}

Abbreviation: DMA, dynamic mechanical analysis.

aTensile strength of self-supporting film.

bElongation of break point in tensile test.

cStorage modulus $\left(E^{\prime}\right)$ at $25^{\circ} \mathrm{C}$

dLoss modulus $\left(E^{\prime \prime}\right)$ at $25^{\circ} \mathrm{C}$.

eGlass transition temperature obtained by DMA.

${ }^{\mathrm{f}} \operatorname{Tan} \delta\left(=E^{\prime \prime} / E^{\prime}\right)$ at glass transition temperature.

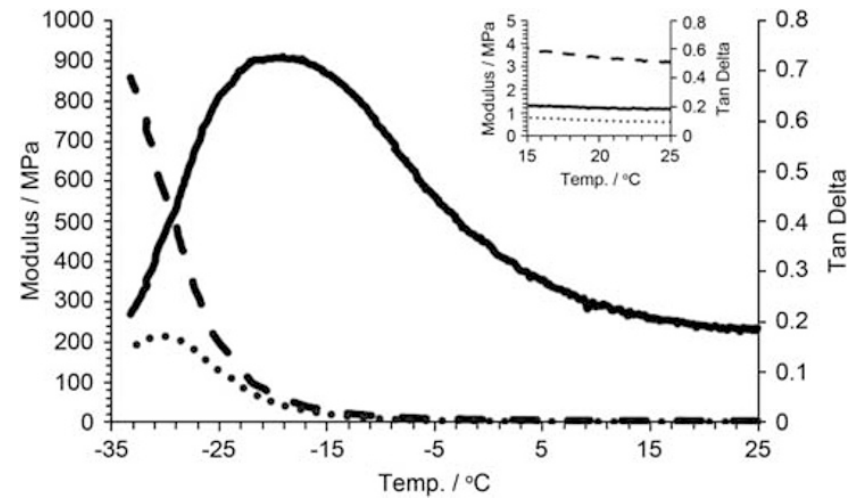

Figure 3 DMA curves of the self-supporting film of derivative 3 ; (- - storage modulus $\left(E^{\prime}\right)$, •• loss modulus $\left(E^{\prime \prime}\right),-\tan \delta=E^{\prime \prime} / E^{\prime}$; single-frequency scanning mode of $1 \mathrm{~Hz}$, cooling rate of $\left.1^{\circ} \mathrm{C} \mathrm{min}^{-1}\right)$.

were also evaluated using tan delta at $T_{\mathrm{g}}$. On the basis of the tan delta values, derivatives $\mathbf{2}$ and $\mathbf{3}$ were seen to have a similar fluidity, whereas derivative $\mathbf{4}$ had low fluidity. This trend is consistent with the elongation percentage values measured during the tensile tests (Table 2). These results suggest that the terminal units have an important role in determining the physical properties of the films.

\section{Chemical oxidation of TTF moieties}

We previously reported the electrochemical properties of the TTF moiety of derivatives 1-3 as measured using cyclic voltammetry. ${ }^{18}$ Derivatives 1-3 were seen to have two reversible redox peaks.

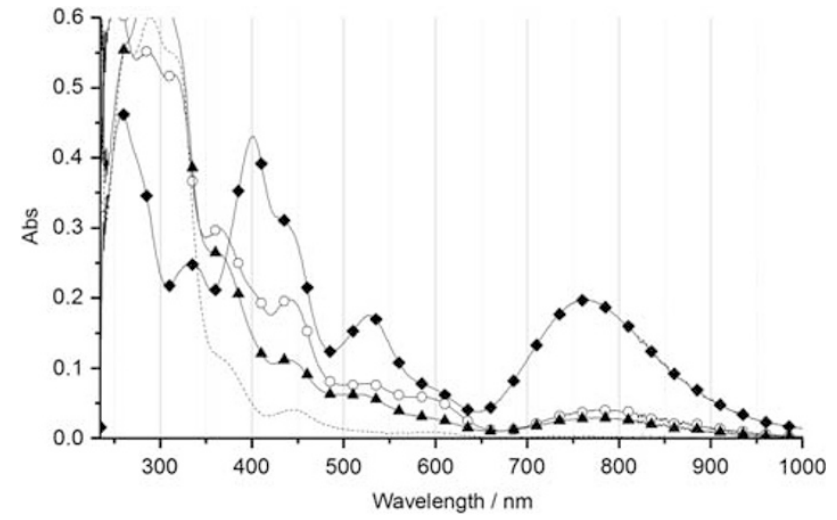

Figure 4 UV-vis absorption spectra of derivative 3 in $0.02 \mathrm{mM} \mathrm{CHCl}_{3}$ solution, and $\mathbf{3}$ with two equivalent of various oxidants; $\mathbf{3}$ (broken line, -- ), $\mathbf{3}+\mathrm{NOBF}_{4}$ (diamond line, -- ), $\mathbf{3}+\mathrm{FeCl}_{3}$ (cycle line, $-\mathrm{O}-$ ) and $\mathbf{3}+\mathrm{I}_{2}$ (triangle line, $-\mathbf{\Lambda}-$ ).

To evaluate the oxidation of the TTF moiety, various chemical oxidations of derivative 3 were carried out in the solution state. $\mathrm{UV}$-vis absorption spectra of $\mathbf{3}$ with various oxidants exhibited the characteristic absorption band of the oxidized TTF moieties (Figure 4). Upon the addition of two equivalents of oxidants to derivative 3, new absorption bands appeared at 580, 530 and $430 \mathrm{~nm}$. The absorption bands were assigned to the mono-oxidized TTF moieties $\left(\mathrm{TTF}^{+} \bullet\right)^{31,32}$ Furthermore, the oxidized form of derivative 3 exhibited a strong absorption band with a maximum at $760 \mathrm{~nm}$. According to the literature, ${ }^{33}$ the UV-vis absorption at around $760 \mathrm{~nm}$ is assigned to a dimer structure of the mono-oxidized TTF moieties. Therefore, the oxidation of derivative 3 caused the formation of a stacked structure of the TTF moieties in solution. As the absorption band at $760 \mathrm{~nm}$ was observed even in a dilute solution, the intramolecular interactions of mono-oxidized TTF moieties were dominant. Owing to the high oxidation potential of nitrosonium tetrafluoroborate $\left(\mathrm{NOBF}_{4}\right)$, the chemical oxidation of TTF moieties proceeded quantitatively, whereas other oxidants such as iron (III) chloride $\left(\mathrm{FeCl}_{3}\right)$ and iodine $\left(\mathrm{I}_{2}\right)$ were less effective.

The stepwise chemical oxidation of derivative 3 with $\mathrm{NOBF}_{4}$ can be monitored by UV-vis spectroscopy (Figure 5). Upon the addition of $\mathrm{NOBF}_{4}$, the absorption band of the oxidized TTF moiety at $760 \mathrm{~nm}$ increased until the amount of oxidant reached two equivalents. The addition of four equivalents of $\mathrm{NOBF}_{4}$ decreased the absorption of the stacked $\left[\mathrm{TTF}^{+} \bullet\right.$, and a new absorption band appeared at $360 \mathrm{~nm}$. This band was assigned to dicationic TTF moieties $\left[\mathrm{TTF}^{2+}\right]^{31,32}$ These results suggest that the decrease in the intensity of the absorption band at $760 \mathrm{~nm}$ is attributed to disaggregation of the stacked TTF moieties because of the repulsive force in $\left[\mathrm{TTF}^{2+}\right]$.

\section{Electrical conductivity}

Table 3 summarizes the electrical conductivity of cast films of $\mathbf{1}, 2$ and 3 under various conditions. ${ }^{18}$ The films exhibited no electrical conductivity in their native state, whereas oxidation of the TTF moieties with 7,7,8,8,-tetracyanoquinodimethane (TCNQ) induced a degree of electrical conductivity in the films (entries 1 and 2).

Derivatives 2 and 3 treated with TCNQ exhibited electrical conductivities of $1.00 \times 10^{-2} \mathrm{~S} \bullet \mathrm{cm}^{-1}$ and $3.66 \times 10^{-2} \mathrm{~S} \bullet \mathrm{cm}^{-1}$, respectively. However, the oxidized film of derivative 1 exhibited no electrical conductivity. When tetracyanoethylene, $\mathrm{I}_{2}, \mathrm{NOBF}_{4}, \mathrm{AgBF}_{4}$, 


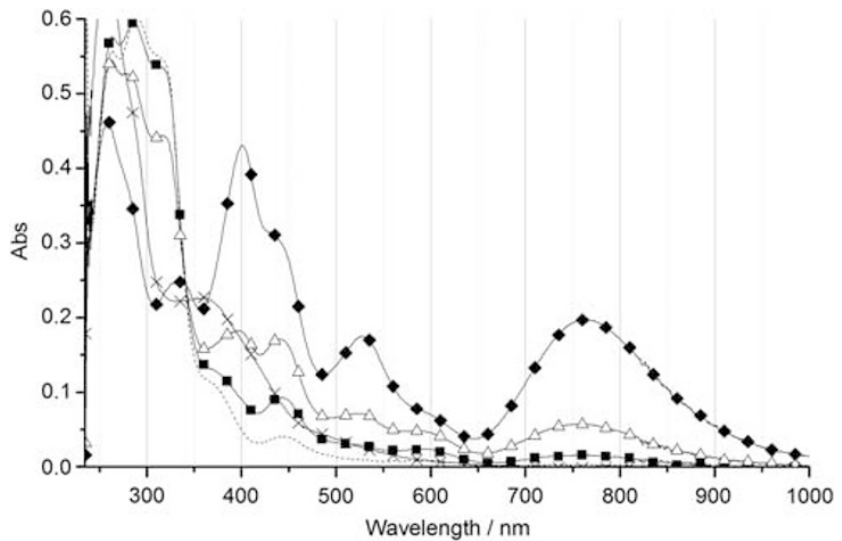

Figure 5 UV-vis absorption spectra of derivative $3(0.02 \mathrm{mM}$ solution in $\mathrm{CHCl}_{3}$ ) with various amounts of $\mathrm{NOBF}_{4} ; 3$ (broken line, -- ), $3+0.5$ eq. $\mathrm{NOBF}_{4}$ (square line, $-\mathbf{-}-$ ), $\mathbf{3}+1.0$ eq. $\mathrm{NOBF}_{4}$ (triangle line, $-\boldsymbol{\Delta}-$ ), $\mathbf{3}+2.0$ eq. $\mathrm{NOBF}_{4}$ (diamond line, $-\longrightarrow$ ) and $\mathbf{3}+4.0$ eq. $\mathrm{NOBF}_{4}$ (cross line, $-x-$ ).

Table 3 Electrical conductivities of derivatives 1-3

\begin{tabular}{|c|c|c|c|c|c|}
\hline \multirow[b]{2}{*}{ Entry } & \multirow[b]{2}{*}{ Oxidant } & \multirow[b]{2}{*}[Ox]{$/[$ derivatives $]$} & \multicolumn{3}{|c|}{ Electrical conductivity $\left(\mathrm{S} \mathrm{cm}^{-1}\right)^{a}$} \\
\hline & & & 1 & 2 & 3 \\
\hline $1^{\mathrm{b}}$ & None & 0 & $N D^{d}$ & ND & ND \\
\hline $2^{b}$ & $\mathrm{TCNQ}$ & 2 & ND & $1.00 \times 10^{-2}$ & $3.66 \times 10^{-2}$ \\
\hline $3^{b}$ & $\mathrm{TCNQ}$ & 1 & ND & $2.51 \times 10^{-3}$ & $9.35 \times 10^{-4}$ \\
\hline $4^{b}$ & $\mathrm{TCNQ}$ & 3 & ND & $1.81 \times 10^{-3}$ & $7.05 \times 10^{-4}$ \\
\hline $5^{b}$ & TCNE & 2 & ND & ND & ND \\
\hline $6^{b}$ & $\mathrm{I}_{2}$ & 2 & ND & ND & ND \\
\hline 7 & $\mathrm{NOBF}_{4}$ & 2 & ND & ND & ND \\
\hline 8 & $\mathrm{FeCl}_{3}$ & 2 & ND & ND & ND \\
\hline 9 & $\mathrm{AgBF}_{4}$ & 2 & ND & ND & ND \\
\hline 10 & $\mathrm{Fe}^{3+\mathrm{c}}$ & 2 & ND & ND & ND \\
\hline
\end{tabular}

Abbreviations: $\mathrm{AgBF}_{4}$, silver tetrafluoroborate; $\mathrm{FeCl}_{3}$, iron (III) chloride; $\mathrm{NOBF}_{4}$, nitrosonium tetrafluoroborate; TCNE, tetracyanoethylene; THF, tetrahydrofuran; TCNQ,

$7,7,8,8$,-tetracyanoquinodimethane.

aElectrical conductivity of the cast film from THF solution was determined by two-probe technique at room temperature.

bPrevious work.

cAcetylferrocenium $\mathrm{BF}_{4}$.

dNot determined due to low electrical conductivity.

$\mathrm{FeCl}_{3}$ and acetylferrocenium tetrafluoroborate $\left(\mathrm{Fe}^{3+}\right)$ were used as oxidants, no electrical conductivity was observed even though the oxidation of TTF moiety could be confirmed by the color change (entries 5-10). These results suggest that the alignment of the TTF moieties of derivatives $\mathbf{2}$ and $\mathbf{3}$ with TCNQ is different from those with the other oxidants in the films.

To investigate the alignment of the TTF moieties, X-ray diffraction measurements of the oxidized cast films were carried out (Figure 6a). As shown in Figure $6 \mathrm{a}$, the TTF moiety of the derivative 3 exhibited no apparent diffraction pattern in a neutral condition. ${ }^{18}$ The observed weak diffraction pattern indicates the presence of a lamellar-like structure constructed by the hydrogen bonding network of the guanosine moieties ${ }^{25,26,34}$ because derivative 4 without a TTF moiety also exhibited a weak diffraction pattern. The diffraction patterns of $\mathbf{3}$ and $\mathbf{4}$ were somewhat different, suggesting different alignments of the hydrogen bonding networks (Supplementary
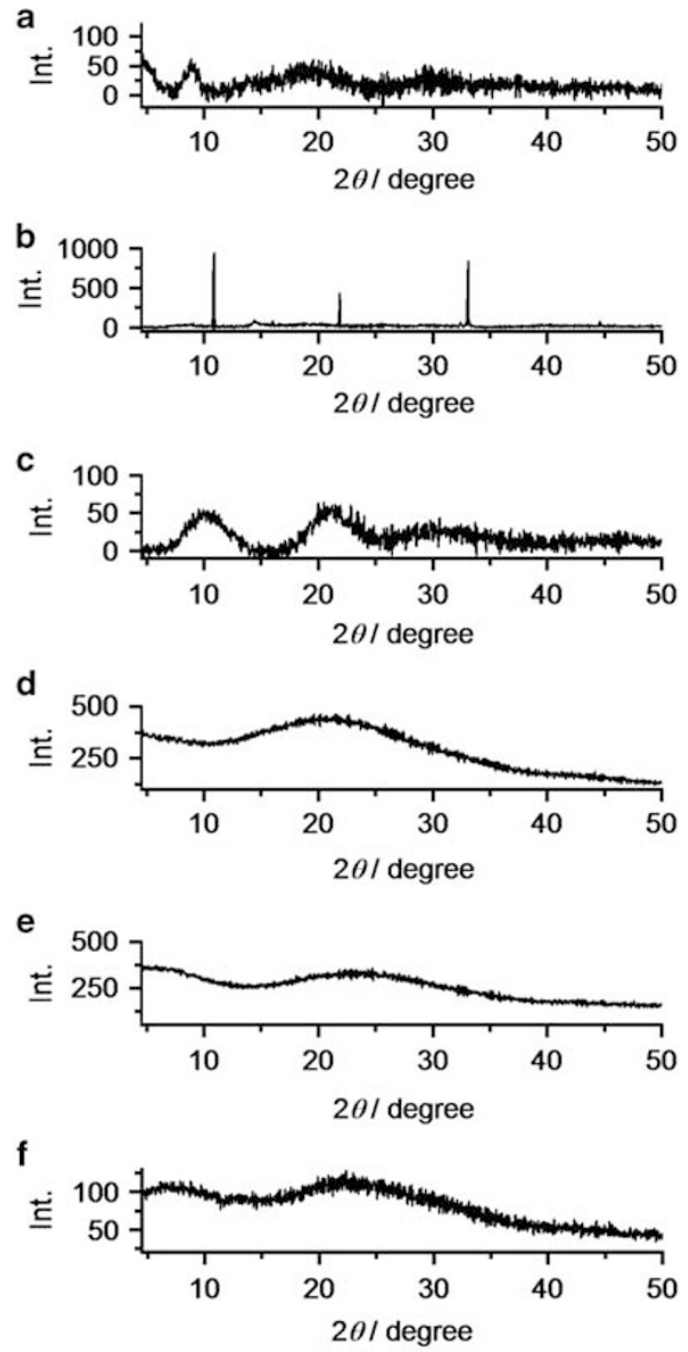

Figure 6 X-ray diffraction patterns of cast films of oxidized derivative $\mathbf{3}$; (a) $3,,^{18}$ (b) $\mathbf{3}+\mathrm{TCNQ}^{18}$ (c) $\mathbf{3}+\mathrm{I}_{2},{ }^{18}$ (d) $\mathbf{3}+2.0$ eq. TCNE, ${ }^{18}$ (e) $\mathbf{3}+2.0$ eq $\mathrm{NOBF}_{4}$ and (f) $\mathbf{3}+2.0$ eq $\mathrm{FeCl}_{3}$.

Figure S4e) ${ }^{34,35}$ In the film of derivative 3 oxidized using TCNQ (entry 2, Table 3$)$, a sharp reflection appeared at $10.9^{\circ}(d=8.19 \AA)$ with higher-order reflections at 21.9 and $33.1^{\circ}$, indicating a high degree of crystallinity (Figure $6 \mathrm{~b}$ ). The sharpness and position of the reflections are consistent with of those of the TTF-TCNQ CT complex in a thin film state. ${ }^{36}$ The film of oxidized derivative 3 is likely to contain similar segregated one-dimensional chain alignment of the TTF moiety and TCNQ molecule. In contrast, the films of derivative 3 oxidized with $\mathrm{I}_{2}$, tetracyanoethylene, $\mathrm{NOBF}_{4}$ or $\mathrm{FeCl}_{3}$ did not exhibit a sharp reflection (Figures $6 \mathrm{c}-\mathrm{f})^{18}$. These results indicate that the TTF moiety and the anion section do not form a periodic structure, such as segregated one-dimensional chains, which is a reasonable explanation for the lack of electrical conductivity (entries $5-8$, Table 3). In the film of 2 oxidized with TCNQ, similar sharp reflections were observed. However, the film of $\mathbf{1}$ oxidized with TCNQ exhibits reflections due to crystals of TCNQ as well as the reflections due to the TTF-TCNQ complex (Supplementary Figure S4). The existence of TCNQ crystals suggests incomplete oxidation of the TTF moiety. Consequently, the electrical conductivity 
of the derivatives in the solid state strongly depends on the periodic alignment of the CT complex of the TTF moiety with TCNQ.

\section{CONCLUSION}

In this work, we synthesized guanosine derivatives with a TTF moiety. The guanosine derivatives formed strong hydrogen bonding networks and could be fabricated into self-supporting films. The films of the derivatives had similar physical properties to those of representative viscoelastic polymers in terms of tensile strength and viscoelasticity, and this was attributed to the hydrogen bonding networks present in the solid state. Both the length and the terminal group of the alkyl chains affected the physical properties. The long alkyl chain of the derivatives is one of the origins of the flexibility of the films. The selfassembled guanosine derivatives with a TTF moiety in the presence of TCNQ underwent one-dimensional alignment of the TTF-TCNQ CT complex in the film state. Taking advantage of this supramolecular approach, electrically conducting films can be fabricated on the macroscale by a simple solution process.

\section{ACKNOWLEDGEMENTS}

This work was partly supported by Grants-in-Aid for Scientific Research from the Ministry of Education, Culture, Sports, Science and Technology, Japan. The Circle for the promotion of science and Engineering is acknowledged for the financial support. The authors are grateful to the Chemical Analysis Center of University of Tsukuba for elemental analyses and NMR spectroscopy. The authors are grateful thank to Mr T Aikawa and Mr J Kanai in TA Instruments for the measurements of DMA. Professor Y Otsuka and Mr Y Shoji are acknowledged for technical supports of the measurements of electrical conductivity.

1 Ferraris, J., Cowan, D. O., Walatka, Jr. V. \& Perlstein, J. H. Electron transfer in a new highly conducting donor-acceptor complex. J. Am. Chem. Soc. 95, 948-949 (1973).

2 Alves, H., Molinari, A. S., Xie, H. \& Morpurge, A. F. Metallic conduction at organic charge-transfer interfaces. Nat. Mater 7, 574-580 (2008).

3 Perlstein, J. H. "Organic Metals"-the intermolecular migration of aromaticity. Angew. Chem. Int. Ed. Engl. 16, 519-534 (1977)

4 Inagi, S., Naka, K. \& Chujo, Y. Functional polymers based on electron-donating TTF and derivatives. J. Mater. Chem. 17, 4122-4135 (2007).

5 Hertler, W. R. Charge-transfer polymers containing 7,7,8,8-tetracyanoquinodimethan and tetrathiafulvalene. J. Org. Chem. 41, 1412-1416 (1976).

6 Green, D. C. \& Allen, R. W. Vinyltetrathiafulvalene. J. Chem. Soc. Chem. Commun. 832-833 (1978).

7 Kaplan, M. L., Haddon, R. C., Wudl, F. \& Feit, E. D. Preparation of some monophenyltetrathiafulvalenes and ( $p$-vinylphenyl)tetrathiafulvalene and its polymerization. J. Org. Chem. 43, 4642-4646 (1978).

8 Frenzel, S., Baumgarten, M. \& Müllen, K. Rod-like tetrathiafulvalene polymers with extended $\pi$-conjugation. Synth. Met. 118, 97-103 (2001).

9 Pittman, Jr. C. U., Ueda, M. \& Liang, Y. Syntheses of poly(urethanes) and poly(sulfonates) containing tetrathiafulvalene nuclei in the backbone. J. Org. Chem. 44, 3639-3642 (1979).

10 Frenzel, S., Arndt, S., Gregorious, R. M. \& Müllen, K. Synthesis of tetrathiafulvalene polymers. J. Mater. Chem. 5, 1529-1537 (1995).

11 Shimizu, T. \& Yamamoto, T. Preparation of a new poly(arylacetylene) with a tetrathiafulvalene (TTF) unit in the side chain. Chem. Commun. 515-516 (1999).

12 lyoda, M., Hasegawa, M. \& Enozawa, H. Self-assembly and nanostructure formation of mulkti-funtional organic $\pi$-donors. Chem. Lett. 36, 1402-1407 (2007).
13 Naka, K., Ando, D., Wang, X. \& Chujo, Y. Synthesis of organic-metal hybrid nanowires by cooperative self-organization of tetrathiafulvalene and metallic gold via chargetransfer. Langmuir 23, 3450-3454 (2007).

14 Kitahara, T., Shirakawa, M., Kawano, S., Bejinn, U., Fujita, N. \& Shinkai, S. Creation of a mixed-valence state from one-dimensionally aligned TTF utilizing the self-assembling nature of a low molecular-weight gel. J. Am. Chem. Soc. 127, 14980-14981 (2005).

15 Kitamura, T., Nakaso, S., Mizoshita, N., Tochigi, Y., Shimomura, T., Moriyama, M., Ito, K. \& Kato, T. Electroactive supramolecular self-assembled fibers comprised of doped tetrathiafulvalene-based gelators. J. Am. Chem. Soc. 127, 14769-14775 (2005).

16 Tatewaki, Y., Hatanaka, T., Tsunashima, R., Nakamura, T., Kimura, M. \& Shirai, H. Conductive nanoscopic fibrous assemblies containing helical tetrathiafulvalene stacks. Chem. Asian. J. 4, 1474-1479 (2009).

17 Hasegawa, M., Enozawa, H., Kawabata, Y. \& Iyoda, M. Hexagonally ordered nanostructures comprised of a flexible disk-like molecule with high self-assembling properties at neutral and cationic states. J. Am. Chem. Soc. 129, 3072-3073 (2007).

18 Choi, S. J., Kuwabara, J. \& Kanbara, T. Electrically Conductive hydrogen-bond-based supramolecular polymer with tetrathiafulvalene moiety: modulation of electrical conductivity and flexibility of film by external stimulus. Chem. Asian J. 5, 2154-2157 (2010)

19 Davis, J. T. \& Spada, G. P. Supramolecular architectures generated by self-assembly of guanosine derivatives. Chem. Soc. Rev. 36, 296-313 (2007).

20 Araki, K. \& Yoshikawa, I. Nucleobase-containing gelators. Top. Curr. Chem. 256, 133-165 (2005)

21 Yoshikawa, I., Li, J., Sakata, Y. \& Araki, K. Design and fabrication of a flexible and selfsupporting supramolecular film by hierarchical control of the interaction between hydrogen-bonded sheet assemblies. Angew. Chem., Int. Ed. 43, 100-103 (2004).

22 Araki, K., Takasawa, R. \& Yoshikawa, I. Design, fabrication, and properties of macroscalesupramolecular fibers consisted of fully hydrogen-bonded pseudopolymerchains. Chem. Commun. 1826-1827 (2001).

23 Gottarelli, G., Masiero, S., Mezzina, E., Pieraccini, S., Rabe, J. P., Samori, P. \& Spada, G. P. The self-assembly of lipophilic guanosine derivatives in solution and on solid surfaces. Chem. Eur. J. 6, 3242-3248 (2000).

24 Spada, G. P., Lena, S., Masiero, S., Pieraccini, S., Surin, M. \& Samorì, P. Guanosine based $\mathrm{H}$-bonded scaffolds: controlling the assembly of oligothiophenes. Adv. Mater 20, 2433-2438 (2008).

25 Yoshikawa, I., Yanagi, S., Yamaji, Y. \& Araki, K. Nucleoside-based organogelators: gelation by the G-G base pair formation of alkylsilylated guanosine derivatives. Tetrahedron 63, 7474-7481 (2007).

26 Takasawa, R., Yoshikawa, I. \& Araki, K. Use of an adjustable soft segment as an effective molecular design for crystal engineering of hydrogen-bonded tape motifs. Org. Biomol. Chem. 2, 1125-1132 (2004).

27 Tanaka, K., Ishiguro, F. \& Chujo, Y. Facile preparation of concentration-gradient materials with radical spin of the mixed-valence tetrathiafulvalene in conventional polymer films. Langmuir 26, 10254-10258 (2010).

28 Bower, D. I. An Introduction to Polymer physics (Cambridge University Press, New York 2002).

29 Menard, K. P. Dynamic Mechanical Analysis: A Practical Introduction. 2nd Edition (CRC Press, Boca Raton, (2008).

30 Gosline, J., Lille, M. \& Carrington, E. Guerette, P., Ortlepp, C. \& Savage, K. Elastic proteins biological role and mechanical properties Phil. Trans. R. Soc. Lond. B 357 , 121-132 (2002).

31 Zhou, Y., Wu, H., Qu, L., Zhang, D. \& Zhu, D. A new redox-resettable molecule-based half-adder with tetrathiafulvalene. J. Phys. Chem. B 110, 15676-15679 (2006).

32 Torrance, J. B., Scott, B. A., Welber, B., Kaufman, F. B. \& Seiden, P. E. Optical properties of the radical cation tetrathiafulvalenium $\left(\mathrm{TTF}^{+}\right)$in its mixed-valence and monovalence halide salts. Phys. Rev. B 19, 730-741 (1979).

33 Ziganshina, A. Y., Ko, Y. H., Jeon, W. S. \& Kim, K. Stable $\pi$-dimer of a tetrathiafulvalene cation radical encapsulated in the cavity of cucurbit[8]uri. Chem. Commun. 806-807 (2004)

34 Yoshikawa, I., Sawayama, J. \& Araki, K. Highly stable giant supramolecular vesicles composed of 2D hydrogen-bonded sheet structures of guanosine derivatives. Angew. Chem. Int. Ed. 47, 1038-1041 (2008).

35 Giorgi, T., Grepioni, F., Manet, I., Mariani, P., Masiero, S., Mezzina, E., Pieraccini, S., Saturni, L., Spada, G. P. \& Gottarelli, G. Gel-like lyomesophases formed in organic solvents by self-assembled guanine ribbons. Chem. Eur. J. 8, 2143-2151 (2002).

36 Fraxedas, J., Molas, S., Figueras, A., Jiménez, I., Gago, R., Auban-Senzier, P. \& Goffman, M. Thin films of molecular metals: TTF-TCNQ. J. Solid State Chem. 168, 384-389 (2002).

Supplementary materialaccompanies the paper on Polymer Journal website (http://www.nature.com/pj) 